\title{
Oxidative stress and dysfunctional NRF2 underlie pachyonychia congenita phenotypes
}

\author{
Michelle L. Kerns, ${ }^{1}$ Jill M.C. Hakim, ${ }^{1}$ Rosemary G. Lu, ${ }^{1}$ Yajuan Guo, ${ }^{1}$ Andreas Berroth, ${ }^{2}$ Roger L. Kaspar, ${ }^{3}$ and Pierre A. Coulombe ${ }^{4,5,6}$ \\ 'Department of Biochemistry and Molecular Biology, Bloomberg School of Public Health, Johns Hopkins University, Baltimore, Maryland, USA. ${ }^{2}$ Department of Pediatrics, Stanford University School of Medicine, \\ Stanford, California, USA. ${ }^{3}$ TransDerm Inc., Santa Cruz, California, USA. ${ }^{4}$ Department of Biological Chemistry, ${ }^{5}$ Department of Dermatology, and ${ }^{6}$ Department of Oncology, School of Medicine, \\ Johns Hopkins University, Baltimore, Maryland, USA.
}

\begin{abstract}
Palmoplantar keratoderma (PPK) are debilitating lesions that arise in individuals with pachyonychia congenita (PC) and feature upregulation of danger-associated molecular patterns and skin barrier regulators. The defining features of PCassociated PPK are reproduced in mice null for keratin 16 (Krt16), which is commonly mutated in PC patients. Here, we have shown that PPK onset is preceded by oxidative stress in footpad skin of $\mathrm{Krt16}^{-/-}$mice and correlates with an inability of keratinocytes to sustain nuclear factor erythroid-derived 2 related factor 2-dependent (NRF2-dependent) synthesis of the cellular antioxidant glutathione (CSH). Additionally, examination of plantar skin biopsies from individuals with PC confirmed the presence of high levels of hypophosphorylated NRF2 in lesional tissue. In $\mathrm{Krt16}^{-/-}$mice, genetic ablation of $\mathrm{Nrf2}$ worsened spontaneous skin lesions and accelerated PPK development in footpad skin. Hypoactivity of NRF2 in Krt16 ${ }^{-/-}$footpad skin correlated with decreased levels or activity of upstream NRF2 activators, including PKC $\delta$, receptor for activated C kinase 1 (RACK1), and p21. Topical application of the NRF2 activator sulforaphane to the footpad of $\mathrm{rrt16}^{-/-}$mice prevented the development of PPK and normalized redox balance via regeneration of CSH from existing cellular pools. Together, these findings point to oxidative stress and dysfunctional NRF2 as contributors to PPK pathogenesis, identify K16 as a regulator of NRF2 activation, and suggest that pharmacological activation of NRF2 should be further explored for PC treatment.
\end{abstract}

\section{Introduction}

Since the genetic etiology of the bullous skin disease epidermolysis bullosa simplex was uncovered in 1991 (1), more than 100 distinct disorders have been shown to arise from or be promoted by mutations in an intermediate filament (IF) gene $(2,3)$. In most instances, these disorders are rare and caused by dominantly acting small mutations that affect the coding sequence of the target IF gene or, more rarely, the splicing of its precursor mRNA. Reflecting the exquisitely tissue-specific and context-dependent regulation of most individual IF genes, these disorders clinically manifest in a very distinctive and typically restricted fashion $(4,5)$. Whereas the pathophysiology underlying IF-based disorders ranges from the relatively simple (e.g., cell/tissue fragility) to the very complex (e.g., premature aging), the development of effective treatments has been lagging owing to a variety of factors including, in part, the plurality of mutations associated with any individual disorder and IF gene $(4,6)$.

Pachyonychia congenita (PC) is a rare condition typified by marked nail dystrophy, palmoplantar keratoderma (PPK), oral lesions, and cystic skin lesions (7).PC is caused by mutations affecting either the type II keratin paralogs KRT6A, KRT6B, or KRT6C or the related type I keratin KRT16 or KRT17 genes $(7,8)$. Consistent with the clinical presentation of PC, these keratin genes are normally expressed in ectoderm-derived epithelial appendages and

Authorship note: J.M.C. Hakim and R.C. Lu contributed equally to this work. Conflict of interest: The authors have declared that no conflict of interest exists. Submitted: September 29, 2015; Accepted: March 24, 2016.

Reference information: / Clin Invest. 2016;126(6):2356-2366. doi:10.1172/JCI84870. in the thicker epidermis of palms and soles, but not in interfollicular epidermis (9). Whereas one observes a pronounced degree of variation in the severity of clinical features across individuals, PPK clearly constitutes the most debilitating aspect of PC and, given the associated pain, has a pronounced if not devastating impact on ambulation and quality of life $(7,10)$. The pathophysiology of PC is in fact quite complex and has perplexed clinicians and biologists alike for years. In part, this likely reflects the fact that K6a, K6b, $\mathrm{K} 16$, and K17 have distinct properties and functions among keratins (5). Further, Krt6a, Krt6b, Krt16, and Krt17 are highly inducible upon disruption of homeostasis in most complex epithelia (9, 11 ), and this property likely adds to the chronic and progressively worsening character of PPK lesions in individuals with PC (12). The poor understanding of the pathophysiology of PPK and PC has unfortunately contributed to delays in devising effective treatments in spite of a well-organized patient- and researcher-based effort to combat this disease, as exemplified in the International Pachyonychia Congenita Consortium (IPCC).

Mice homozygous for a null allele at the Krt16 locus develop a PC-like presentation that includes, in those animals surviving perinatal lethality, the development of spectacular PPK lesions in a completely penetrant fashion by 6 weeks of age (13). Calluses arise preferentially at sites of mechanical pressure in the footpads of $\mathrm{Krt16}^{-/-}$mice and otherwise feature all of the hallmarks of PC-related PPK, including modest hyperproliferation along with massive thickening of the living epithelium and stratum corneum layers of the epidermis (referred to as hyperkeratosis) $(7,13)$. Interestingly, established PPK lesions in $\mathrm{Krt16}^{-/-}$mice show little evidence of cell 
A

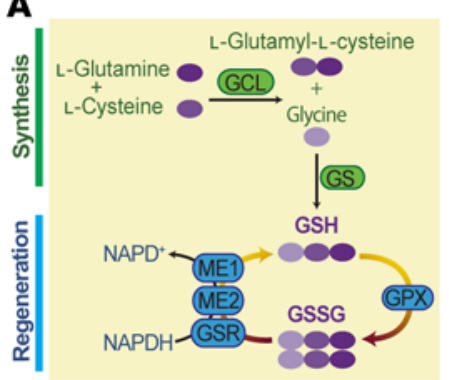

E

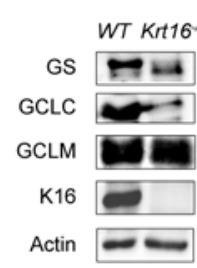

B
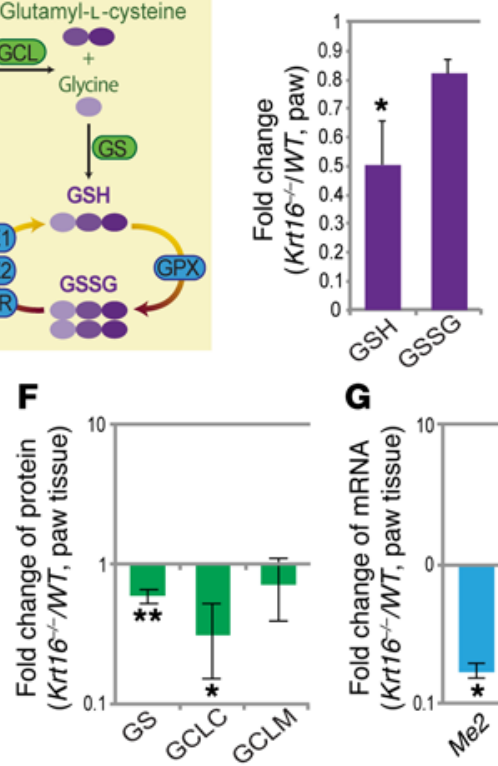

G

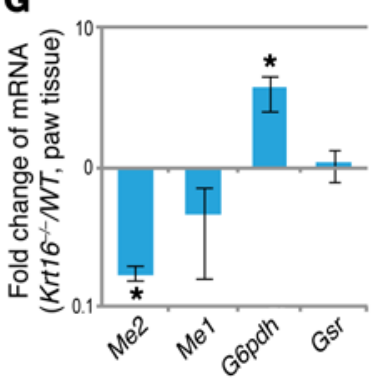

D
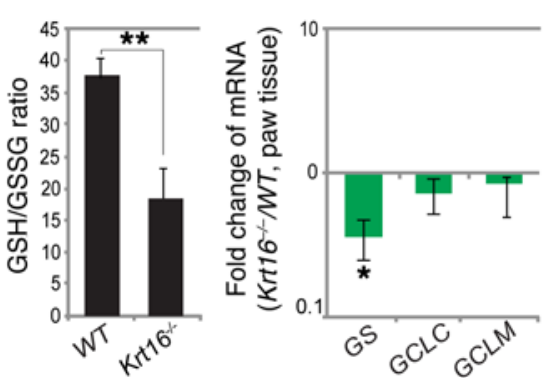

H

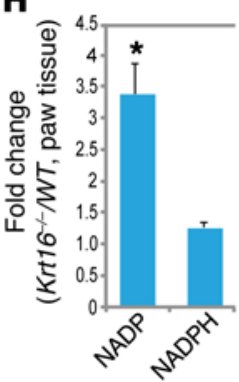

Figure 1. Decrease in GSH synthesis in $\mathrm{Krt16}^{-/-}$prelesional paw skin. (A) Schematic representation of GSH synthesis and metabolism. (B) Fold change in GSH and GSSG for $\mathrm{Krt16}^{-/-}$relative to WT. (C) GSH to GSSG ratio for $\mathrm{Krt16}^{-1-}$ and WT paw skin. For $\mathbf{B}$ and $\mathbf{C}$, data represent mean \pm SEM of 6 biological replicates, Student's $t$ test. (D) Relative fold change in mRNA levels of GSH synthesis enzymes. Data represent mean \pm SEM of 4 to 6 biological replicates. Student's $t$ test. (E) Representative Western blot of GSH synthesis enzymes and K16 with actin as loading control. (F) Histogram of quantitation of Western blot data. Data represent mean \pm SEM of 3 biological replicates and are normalized to actin. Student's $t$ test. (C) Relative fold change in mRNA levels of GSH metabolism enzymes. Data represent mean \pm SEM of 4 to 6 biological replicates. Student's $t$ test. For $\mathbf{D}, \mathbf{F}$, and $\mathbf{G}$, log scale. (H) Relative fold change of total NADP and NADPH. Data represent mean \pm SEM of 6 biological replicates involving 1-month-old male mice. Student's $t$ test. ${ }^{*} P<0.05$; ${ }^{* *} P<0.01$.

fragility (11). A follow-up effort showed that footpad lesions from $\mathrm{Krt16}^{-/-}$mouse paw skin and biopsies from clinically involved plantar skin in individuals with PC show a remarkably similar molecular signature whereby the expression of genes coding for danger-associated molecular patterns (DAMPs), e.g., alarmins, and regulators of skin barrier formation, is markedly elevated (11). This effort also showed that subjecting clinically uninvolved skin in $\mathrm{Krt}^{-/-}$mice to wounding or topically applied chemical irritants results in an exaggerated upregulation of DAMP/alarmin and skin barrier genes relative to controls and, further, that Krt16 is an integral part of a strong genetic network involving such genes (11). Here, we provide compelling evidence that K16 regulates the function of nuclear factor erythroid-derived 2 related factor 2 (NRF2), a master regulator of cellular responses to oxidative stress and of epidermal homeostasis, suggesting a new avenue for the therapeutic management of PC-associated PPK lesions.

\section{Results}

Impaired glutathione synthesis in prelesional $\mathrm{Krt}^{-/-}$front paw tissue. Glutathione (GSH) is the most abundant endogenous antioxidant in cells and is crucial for the maintenance of cellular redox balance (14). GSH prevents damage from ROS in part by consuming ROS through a reduction reaction that converts reduced GSH to its oxidized state (glutathione disulfide [GSSG]). The ratio of GSH to GSSG is routinely used as a measure of cellular toxicity (15). GSH is a tripeptide ( $\gamma$-L-glutamyl-L-cysteinylglycine) synthesized through a 2-step process catalyzed by the enzymes glutamine cysteine ligase (GCL) and glutathione synthetase (GS). GCL is a heterodi- mer that catalyzes the initial rate-limiting step of GSH synthesis and is composed of an essential catalytic subunit (GCLC) (16) and a modulatory subunit (GCLM) that enhances the efficiency of GCL, but is not required for function (17). GSH can also be regenerated from GSSG in a reaction that is catalyzed by the enzyme glutathione reductase (GSR) and utilizes the coenzyme NADPH as an electron donor (18). In turn, levels of NADPH are replenished by the action of glucose-6-phosphate dehydrogenase (G6PD) and malic enzymes 1 and 2 (ME1/2). The levels and activities of each of these enzymes (see Figure 1A) contribute to set the cellular levels of GSH and GSSG and the resulting GSH/GSSG ratio.

We previously reported that pharmacological manipulation of GSH levels upregulates Krt16 expression in murine epidermis (19). Here, we set out to characterize the redox balance in prelesional paw skin of 1-month-old male $\mathrm{Krt16}^{-/}$mice. Relative to control, $\mathrm{Krt16}^{-/-}$paw tissue had a significant 2-fold decrease in GSH levels and slightly reduced GSSG levels (Figure 1B). The net effect was a lower GSH to GSSG ratio $\left(18.5 \pm 4.7\right.$ for $\mathrm{Krt16}^{-/}$paws versus $37.9 \pm$ 3.3 for WT control), indicative of more oxidative stress in $\mathrm{Krt}^{-1} 6^{-1}$ skin relative to WT skin (Figure 1C). To test whether the decline in GSH in $\mathrm{Krt16}^{-/-}$paw skin was due to alterations in de novo synthesis or regeneration, we assessed the expression of relevant enzymes. There was a notable $2.8 \pm 0.1$-fold drop in GS mRNA (Figure 1D; mean \pm SEM) and markedly less GS and GCLC protein relative to control (Figure 1, E and F). In contrast, we found no remarkable difference in Gsr mRNA (Figure 1G). Although the mRNA levels of $M e 2$ and G6pd were decreased $5.3 \pm 0.2$-fold and increased 3.7 \pm 0.9 -fold, respectively, the overall levels of NADPH were similar 
A

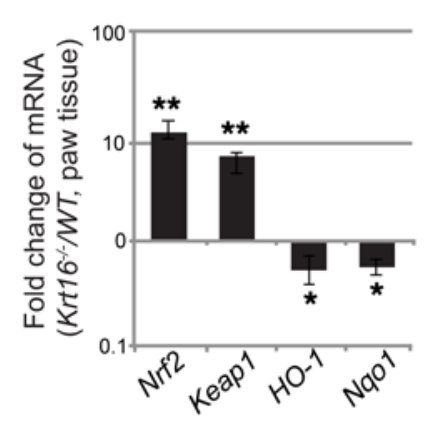

C
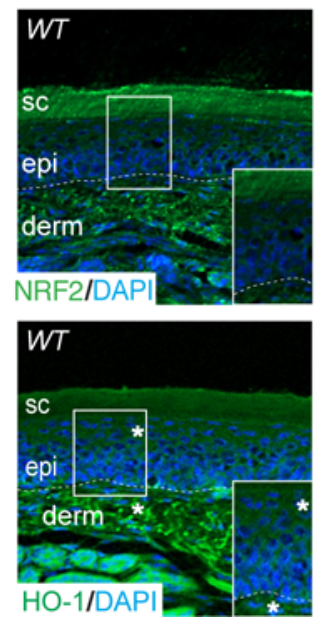

B

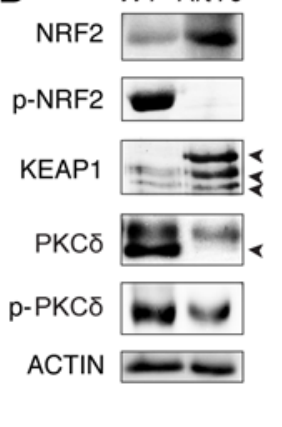

D
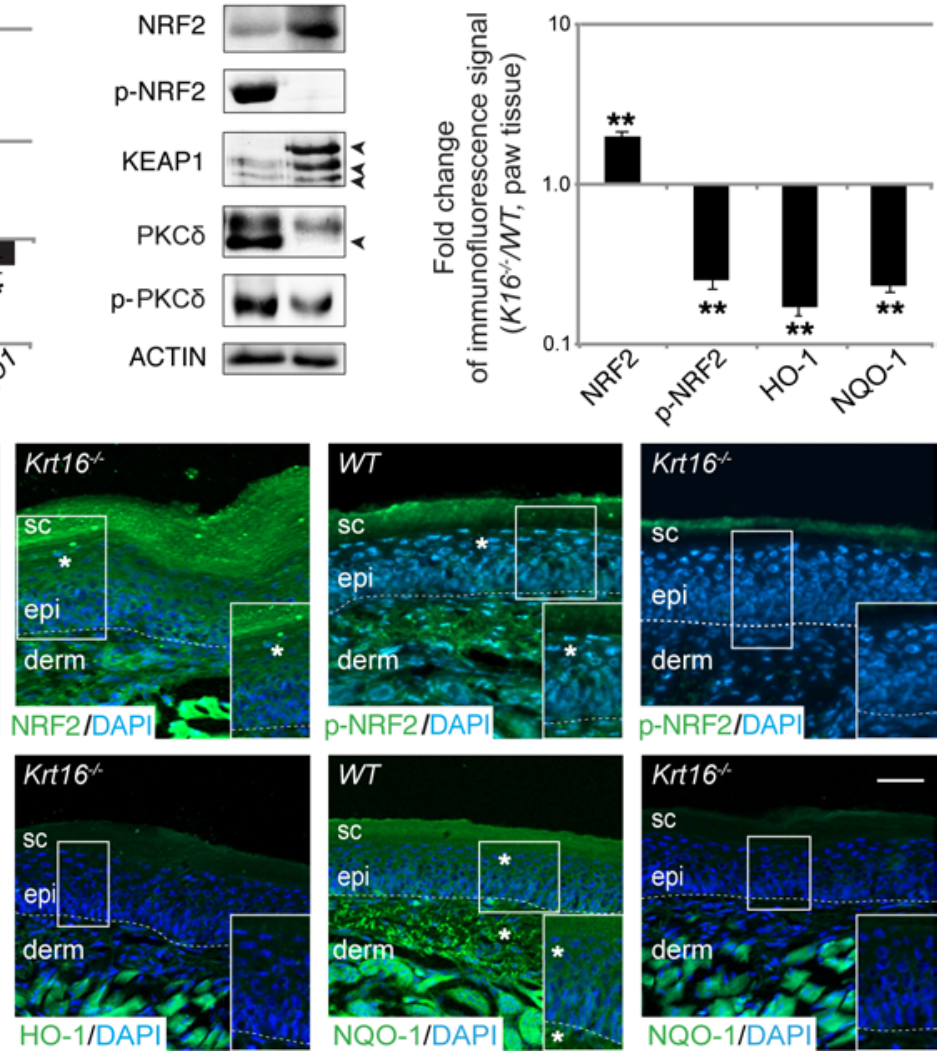

Figure 2. Impact of Krt16 expression on NRF2 activation. (A) Relative fold change of mRNA for Nrf2, Keap1, HO-1, and Nqo1 in prelesional 1-month-old male $\mathrm{Krt16}^{-1-}$ paw skin relative to WT controls. Data represent mean \pm SEM of 4 to 6 mice. Student's $t$ test. (B) Representative Western blot for NRF2, p-NRF2, KEAP1, PKC $\delta$, and p-PKC $\delta$ of 3 experiments, with actin as loading control. Arrowheads denote the 3 bands of KEAP1 and the lower band that represents PKC $\delta$. (C) Indirect immunofluorescence for NRF2, p-NRF2, HO-1, and NQO-1. DAPI, nuclear staining; sc, stratum corneum; epi, epidermis; derm, dermis. Dotted lines mark the dermoepidermal junction. Scale bars: $50 \mu \mathrm{m} ; 100 \mu \mathrm{m}$ (inset). Asterisks mark areas of increased immunofluorescence signal. (D) Quantitation of immunofluorescence signal for WT and $\mathrm{Krt16}^{-/-}$paw tissue stained for NRF2, p-NRF2, HO-1, and NQO-1. Data represent mean \pm SEM of 101 -month-old mice, 2 images per mouse. Student's $t$ test. ${ }^{*} P<0.05 ;{ }^{* *} P<0.01$.

to those of control (Figure $1 \mathrm{H}$; mean $\pm \mathrm{SEM}$ ). Taken together, these findings suggest that GSH synthesis is impaired in murine paw tissue lacking Krt16, creating a state of oxidative stress prior to the onset of hyperkeratotic lesions.

Lowering GSH levels causes PPK-like alterations in WT mouse skin. To gauge the effect of enhanced oxidative stress alone on skin morphology, we next treated 1-month-old WT male mice through i.p. injections of $50 \mu$ mole l-buthionine-sulfoximine (BSO), an inhibitor of GSH synthesis, twice weekly. Levels of GSH and GSSG levels in paw tissue were assessed, and histological analysis was performed after 4 weeks of treatment. Relative to vehicle control, systemic BSO resulted in a significant decrease of both GSH and GSSG in paw tissue (Supplemental Figure 1A; supplemental material available online with this article; doi:10.1172/ JCI84870DS1). The ratio of GSH to GSSG remained within normal limits (Supplemental Figure 1B). Although BSO treatment did not cause any obvious macroscopic changes of the paws (Supplemental Figure 2C), it resulted in a significant, $1.8 \pm 0.1$-fold, increase of epidermal thickness (Supplemental Figure 1, D and E; mean \pm SEM) along with the appearance of marked, PPK-like downward epidermal projections, which is quantitatively reflected through a measurement of the relative length of the dermoepi- dermal interface (Supplemental Figure 1, D and F). These findings establish that even a time-limited state of oxidative burden suffices to induce epidermal changes related to PPK lesions and lend strong support for their potentially important influence upon the pathogenesis of PC-related PPK.

The transcription factor NRF2 is expressed but hypoactive in lesional $\mathrm{Krt16}^{-/-}$footpad skin. NRF2 regulates a transcriptional program that preserves cellular redox homeostasis and includes a myriad of GSH synthesis and metabolism genes. NRF2 acts through antioxidant response elements (AREs) that are located in the promoter regions of these genes (20). The promoter region of $\mathrm{Krt16}$ contains a functional ARE (21). We hypothesized that K16 may itself contribute to NRF2 activity in the setting of a positive feedback loop and that the defect in GSH synthesis observed in $\mathrm{Krt16}^{-/-}$paw tissue reflects a malfunctioning of the NRF2 signaling pathway.

We first evaluated the status of NRF2/KEAP1 signaling ahead of the development of PPK-like lesions in footpad skin of $\mathrm{Krt16}^{-/}$ mice. Surprisingly, loss of $K r t 16$ caused a $13.2 \pm 2.4$-fold increase of Nrf2 mRNA as well as a 2 -fold increase in NRF2 protein in paw tissue of 1-month-old $\mathrm{Krt16}^{-/-}$male mice (Figure 2, A-D; mean \pm SEM). In striking contrast, the mRNA levels of 2 classical NRF2 target genes, heme oxygenase 1 (HO-1) and NAD(P)H dehydroge- 
A

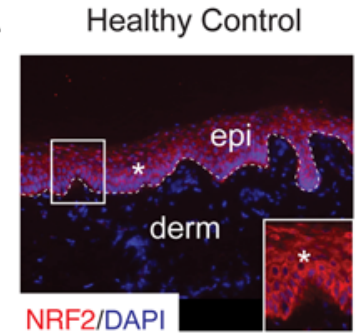

B

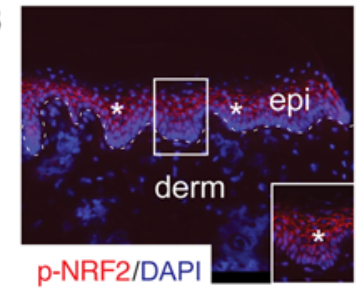

C

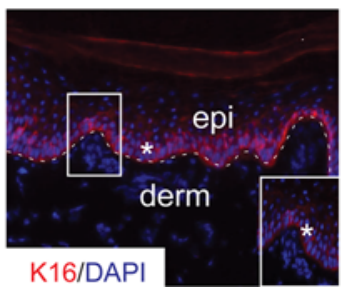

KRT16 N125D PC Patient
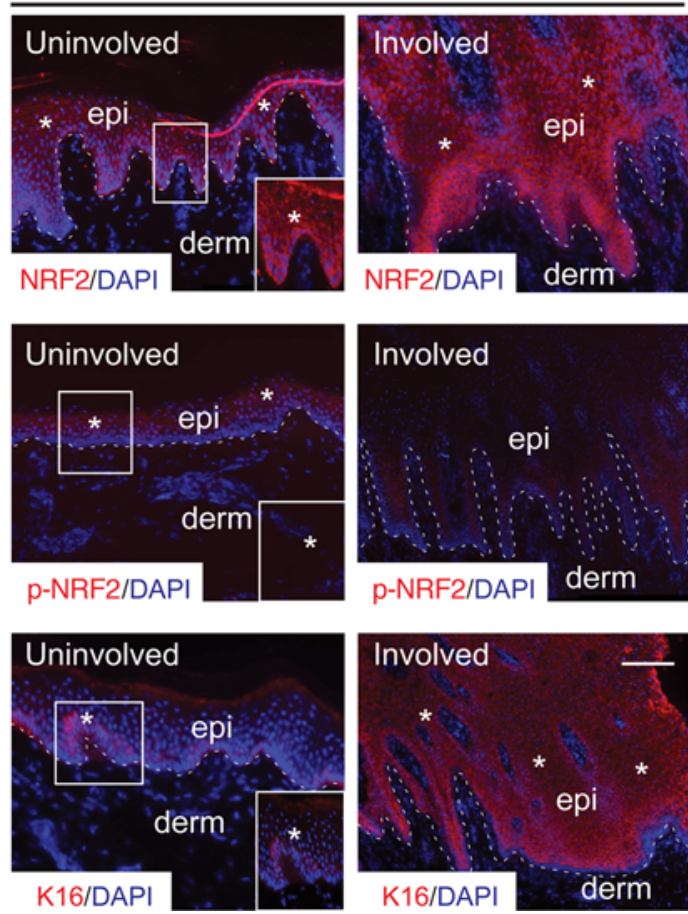

Figure 3. Status of NRF2 in KRT16 N125 deletion PC patient samples. Indirect immunofluorescence for NRF2 (A), p-NRF2 (B), and K16 (C) in healthy control and PC patient skin. Scale bar: $50 \mu \mathrm{m} ; 200 \mu \mathrm{m}$ (inset). Asterisks mark areas of increased immunofluorescence. Dotted lines mark the dermoepidermal junction. Uninvolved, nonlesional skin; involved, lesional skin.

nase quinone 1 (Nqo1) $(22,23)$, were approximately 2 -fold lower in $\mathrm{Krt16}^{-/-}$relative to control paw skin (Figure 2A). This corresponded with $5.9 \pm 0.2$-fold and 6.7 \pm 0.4 -fold decreases in the immunofluorescence signal for HO-1 and NQO-1 in $\mathrm{Krt16}^{-/-}$epidermis relative to control (Figure 2, C and D). Taken together with the marked reduction in GSH synthesis (Figure 1), these findings imply that, although NRF2 transcript and protein levels are increased, there is an impairment of the NRF2 signaling pathway in 1-month-old $\mathrm{Krt16}^{--}$paw skin, prior to the development of PPK lesions.

We assessed whether the loss of K16 could be impeding NRF2 activation through KEAP1, the cytoplasmic inhibitor that normally binds to NRF2 and promotes its proteosomal degradation (24). Keap1 mRNA levels were $7.7 \pm 1.7$-fold higher in $\mathrm{Krt16}^{-/-}$paw skin (Figure $2 \mathrm{~A})$, and the levels of KEAP1 protein were also elevated approximately 2-fold (Figure 2B). This said, the occurrence of high levels of NRF2 protein imply that the reduction of NRF2 activity is unlikely solely due to altered function of KEAP1 in the absence of K16.

$\mathrm{PKC} \delta$-mediated phosphorylation of NRF2 at serine 40 is required for release of NRF2 from KEAP1 and its subsequent stabilization and nuclear translocation (25), affording an additional opportunity to assess NRF2 activity status. Phosphorylated NRF2 (p-NRF2) (Ser40) was decreased in $\mathrm{Krt16}^{-/-}$skin, as detected by both Western blotting (Figure 2B) and indirect immunofluorescence (Figure 2, C and D). Protein levels of total PKC $\delta$ and PKC $\delta$ phosphorylated at Thr505, which is located in the activation loop, were also reduced in $\mathrm{Krt16}^{-/-}$paw tissue relative to control (Figure 2D). Total PKC $\delta$ and p-PKC $\delta$ (Thr505) levels were down $2.7 \pm 0.2$-fold and $2.9 \pm 0.3$-fold, respectively, in $\mathrm{Krt}_{16^{-/-}}$paw tissue relative to control (mean $\pm \mathrm{SEM} ; n=3$ ). Together, these findings establish that the transcription factor NRF2 is present in abundance, but dysfunctional in prelesional footpad skin of $\mathrm{Krt16}^{-1}$ mice, correlating with impaired expression and activity of its upstream regulator $\mathrm{PKC} \delta$.

NRF2 is present but hypoactive in lesional plantar epidermis of individuals with PC. To test the clinical applicability of our findings in the $\mathrm{Krt16}^{-/-}$mouse model, we evaluated NRF2 and p-NRF2 expression in plantar skin of individuals suffering from PC. Genetically, the PC samples surveyed included a KRT16N125D mutation (Figure 3), a KRT6cN172D mutation (Supplemental Figure 2), a KRT6aD432_E470dup mutation (data not shown), and a KRT6a N172del mutation (data not shown).

In all $4 \mathrm{PC}$ cases as well as the plantar skin of a healthy volunteer, substantial expression of NRF2 can be detected in plantar epidermis via indirect immunofluorescence, irrespective of the presence of lesions. By contrast, expression of p-NRF2 is markedly lower in the lesional epidermis of individuals with PC when compared with clinically normal-appearing skin from a proximal area in the same individual's plantar skin (e.g., compare "involved" and "uninvolved" in Figure 3B) and in plantar epidermis from a healthy volunteer (Figure 3B; see also Supplemental Figure 2). Consistent with a previous study (8), expression of K16 was also increased in lesional skin tissue from the individual with KRT16N125D PC relative to plantar skin of a healthy volunteer (Figure $3 \mathrm{C}$ ). These observations corroborate the findings in the $\mathrm{Krt16}^{-/-}$mouse model and support a possible association between PPK lesions and hypoactive NRF2. They further suggest that impaired NRF2 activity is not restricted to cases of PC 
A
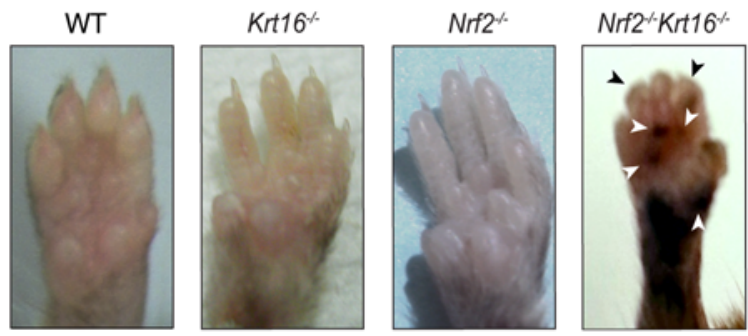

B
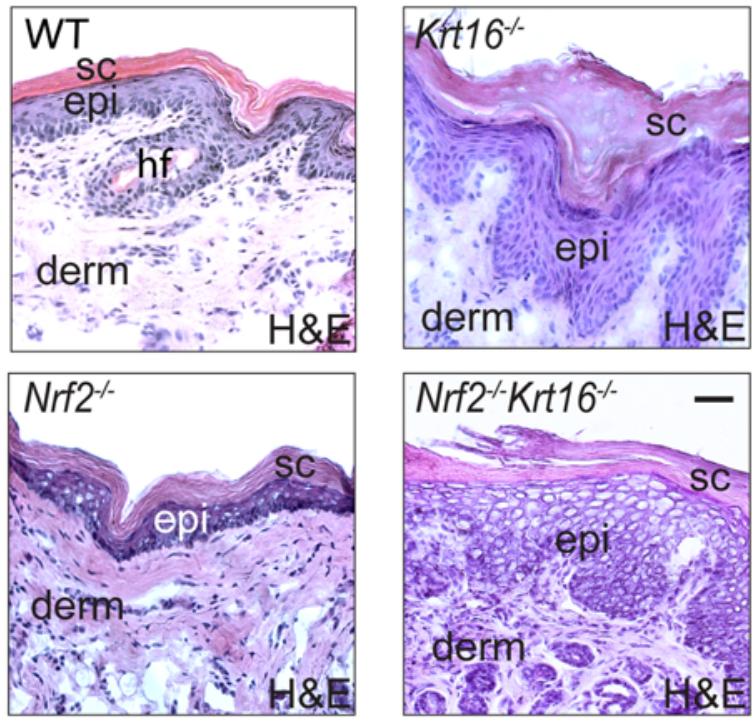

C

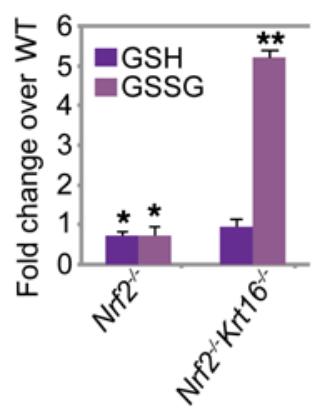

D

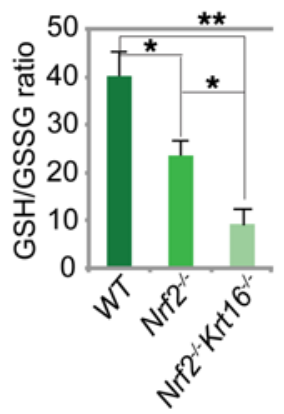

arising from mutations in KRT16, though a full exploration of this important issue will require studies on additional PPK/PC biopsies.

Substantiating the link between K16 and NRF2 activation. We next conducted several experiments to test the hypothesis that Krt16/ K16 plays a significant role in regulating NRF2 activity in skin keratinocytes. First, this hypothesis predicts that the $\mathrm{Krt}^{-16^{-/}}$skin phenotype should worsen in the setting of a complete NRF2 deficiency. To test this prediction, we mated the $\mathrm{Krt16}^{-1-}$ mice with $\mathrm{Nrf2} 2^{---}$mice (available in the same genetic background) and assessed the onset of paw lesions and overall skin appearance in the resulting offspring. Representative images of paws from $\mathrm{Krt16}^{--}$, $\mathrm{Nrf2} 2^{--}$, and $\mathrm{Nrf2} 2^{--} \mathrm{Krt16}^{--}$mice are shown in Figure 4A. Of the $\mathrm{Nrf2}^{2-} \mathrm{Krt16}^{-}$ mice that were monitored $(n=20)$, approximately $50 \%$ died by $\mathrm{P} 1$ and the remaining 50\% developed hyperkeratotic calluses and digital contractures by 3 weeks of age. This is 3 weeks earlier than the average age of onset for clinically evident paw lesions in $\mathrm{Krt16}^{-1}$

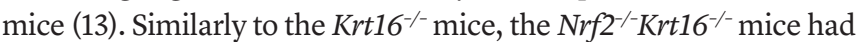
thickened epidermis and stratum corneum at 1 month of age. Like
Figure 4. Loss of NRF2 results in earlier onset of hyperkeratotic front paw calluses in $\mathrm{Krt16}^{-/-}$mice. Images (A) and H\&E staining (B) of representative

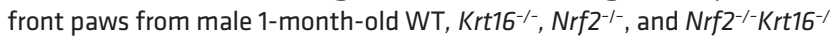
mice. hf, hair follicle. White arrows mark hyperpigmented calluses; black arrowheads mark contracted digits. Scale bar: $50 \mu \mathrm{m}$. (C) Fold change in GSH and GSSG for $\mathrm{Nrf2}^{-1-}$ and $\mathrm{Nrf2}^{-1-} \mathrm{Krt16}^{-/-}$paw skin relative to WT. Data represent mean \pm SEM of 6 biological replicates. Student's $t$ test. (D) GSH/ GSSG ratio for WT, $\mathrm{Nrf2}^{-1-}$, and $\mathrm{Nrf2}^{-/-} \mathrm{Krt16}^{-/-}$paw skin. Data represent mean \pm SEM of 6 biological replicates. ANOVA. ${ }^{*} P<0.05 ;{ }^{* *} P<0.01$.

the $\mathrm{Nrf2}^{-/}$mice, the $\mathrm{Nrf2}^{2--\mathrm{Krt16}} 6^{-/}$mice also exhibited striking vacuolar changes in the epidermis (Figure 4B). Moreover, at 1 month of age, the ventral surfaces of $\mathrm{Nrf}^{-}-\mathrm{Krt16}-\mathrm{C}^{-}$mice were remarkable for areas of hyperkeratosis, alopecia, and extensive eschar (Supplemental Figure 3). The dorsal surfaces of the $\mathrm{Nrf2}^{-1-} \mathrm{Krt} 16^{-/-}$mice also exhibited thinning hair and patches of erythema (Supplemental Figure 3). These findings establish the existence of a functional link between K16 and NRF2 in skin keratinocytes. We also assessed the levels of GSH and GSSG in paw tissue taken from 1-month-old $\mathrm{Nrf2} 2^{-/}$and $\mathrm{Nrf2}^{-1-\mathrm{Krt}} 16^{-/-}$male mice. GSH and GSSG levels, as well as the GSH to GSSG ratio, were decreased in $\mathrm{Nrf2}^{--}$paw skin, which indicates increased oxidative stress relative to WT paw skin. The loss of both Nrf2 and Krt16 has an even more dramatic impact on redox balance. GSH is $1.4 \pm 0.23$-fold lower in $\mathrm{Nrf2}^{-/-} \mathrm{Krt16}^{-/}$paw skin, but GSSG is approximately 5 -fold higher than WT (Figure 4C; mean \pm SEM). There was also significant effect of genotype on the GSH to GSSG ratio (ANOVA, $P=0.0001$ ). In the absence of both $N r f 2$ and $K r t 16$, the GSH to GSSG ratio dropped to a remarkably low $9.08 \pm 3.58$ relative to $40.1 \pm 5.2$ and $23.5 \pm 3.2$ for WT and $N r f 2^{--}$, respectively (Figure $4 \mathrm{D}$; mean \pm SEM).

Second, this hypothesis predicts that expression of K16 in cultured skin keratinocytes should enhance NRF2 activity. To test this prediction, we transfected immortalized 308 mouse skin keratinocytes that have low endogenous Krt16 expression with construct encoding either GFP-fused K16 or GFP-fused K14, used here for comparison, and evaluated the subcellular localization and phosphorylation status of NRF2 by indirect immunofluorescence. Marked increases in nuclear-localized NRF2 and p-NRF2 (by 2.2. \pm 0.75 -fold and $5.3 \pm 0.48$-fold, respectively) were detected in cells expressing GFP-K16 relative to those expressing GFPK14 (Figure 5 , A and B; $n=40$ cells per group, mean \pm SEM). These findings suggest that, relative to K14, K16 exhibits a significantly stronger ability to enhance NRF2 function in skin keratinocytes.

Third, we probed for a potential mechanism through which K16 could influence PKC $\delta$ expression and activation in cultured skin keratinocytes. The receptor for activated C kinase 1 (RACK1) has been shown to stabilize the active conformation of PKCs, including the PKC $\delta$ isoform, and act as a shuttling protein (26). Intriguingly, K5 and K14 have been shown by others to physically interact with RACK1, leading to its sequestration and the inhibition of PKC $\alpha$ activity (27). We hypothesized that, unlike the basalspecific K5/K14, K16 would not interact with and/or sequester RACK1 in keratinocytes. Consistent with previous findings (27), substantial colocalization of RACK1 and K14 was observed in 308 mouse skin keratinocytes in culture. In contrast, faint or no colocalization of RACK1 and K16 was detected in 308 cells that were transfected with GFP-fused K16 (Figure 5C). 
A
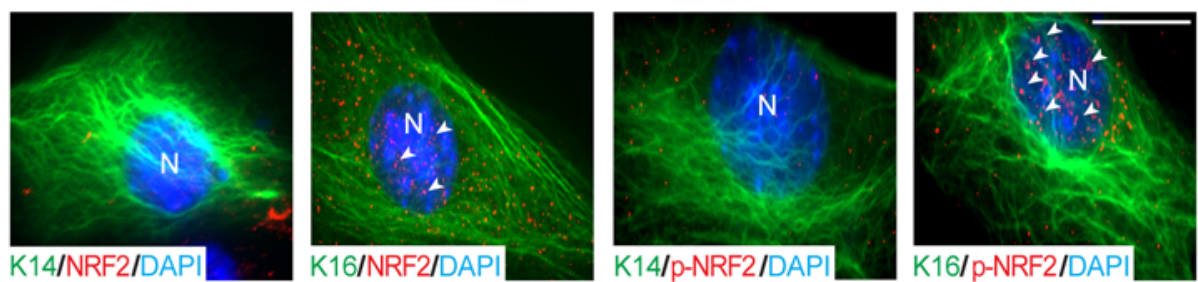
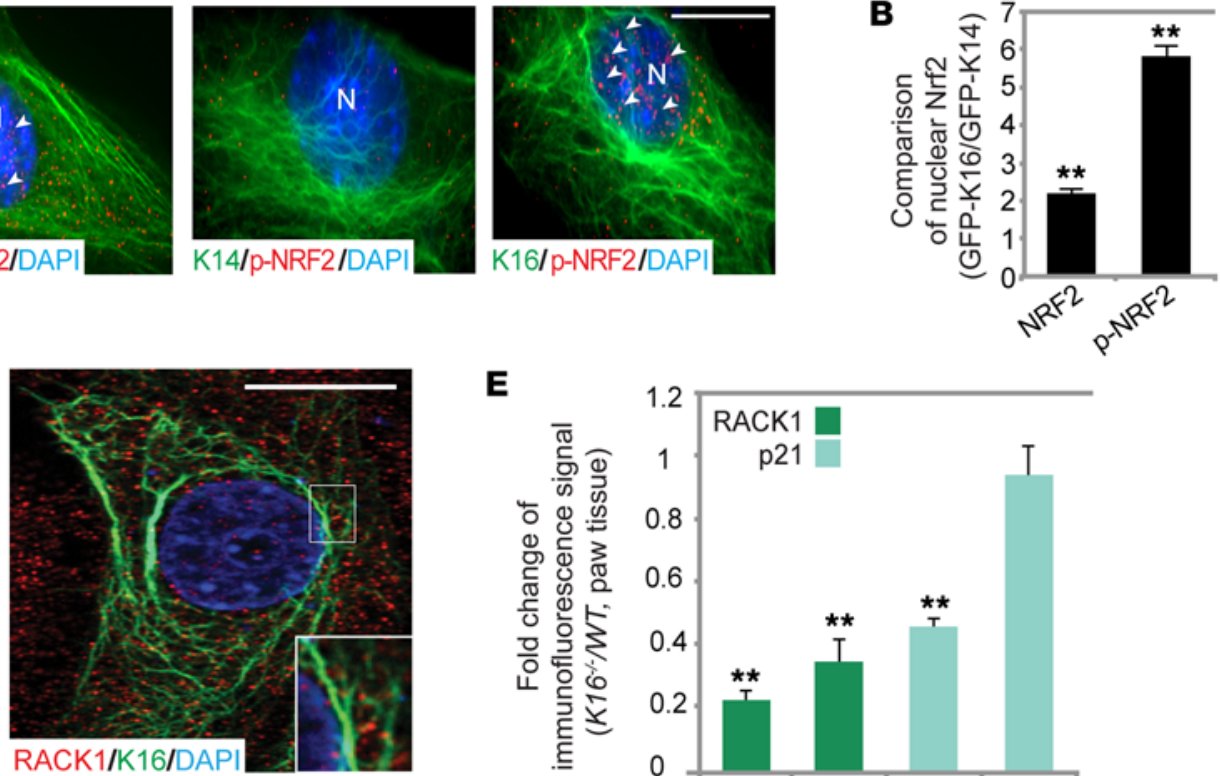

RACK1/K14/DAP

C

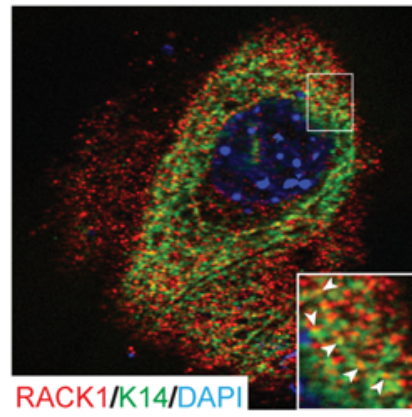

D
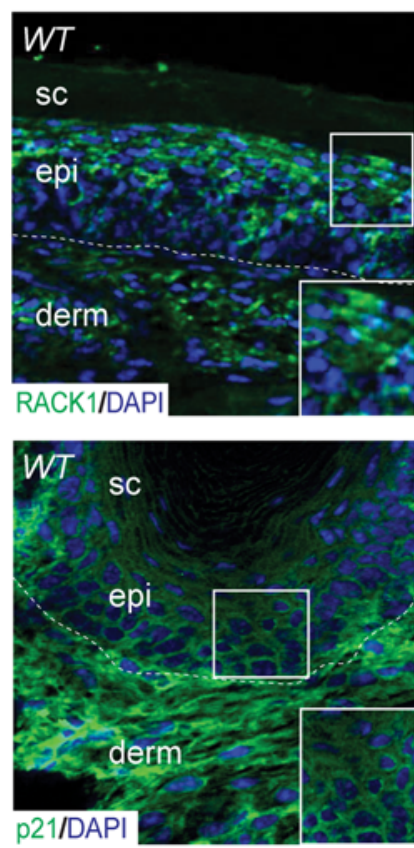
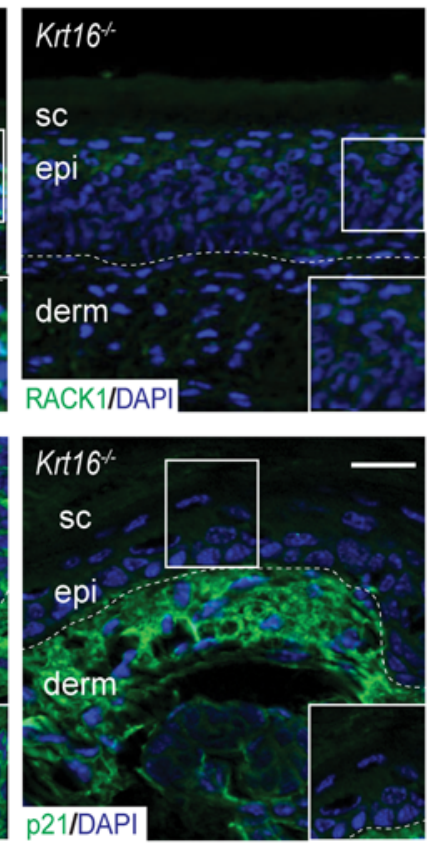

E

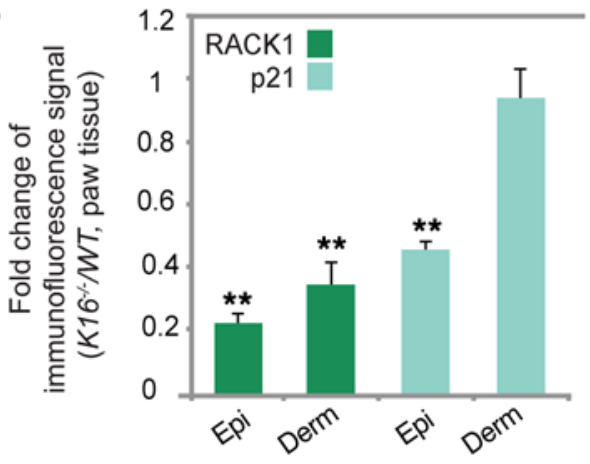

F

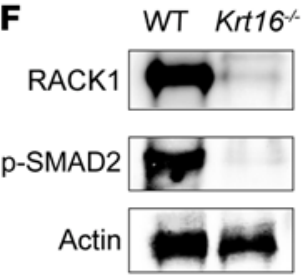

H

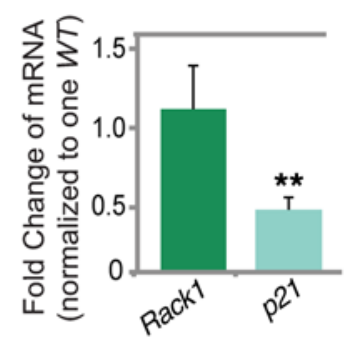

Figure 5. A link between Krt16 and NRF2 activation. (A) Indirect immunofluorescence for NRF2 and p-NRF2 in 308 cells transfected with GFP-fused K14 or K16. Scale bar: $10 \mu \mathrm{m}$. Arrowheads mark increased nuclear immunofluorescence signal. N, nucleus. (B) Quantitation of intranuclear NRF2 and p-NRF2 immunofluorescence signal. Data represent mean \pm SEM for 40 cells per group normalized for nuclear size. Student's $t$ test. (C) Indirect immunofluorescence for RACK1 in 308 cells transfected with GFP-fused K16. Scale bar: $10 \mu \mathrm{m} ; 100 \mu \mathrm{m}$ (inset). (D) Indirect immunofluorescence for RACK1 and p21 in paw skin of 1-month-old male mice. Dotted lines mark dermoepidermal junction. Scale bar: $50 \mu \mathrm{m} ; 100 \mu \mathrm{m}$ (inset). (E) Relative fold change of immunofluorescence signal for WT and $\mathrm{Krt16}^{-/-}$paw tissue stained for RACK1 and p21. Data represent mean \pm SEM of 10 mice, 2 images per mouse. Student's $t$ test. (F) Representative Western blot for RACK1 and p-SMAD2 of 3 experiments with actin as loading control. (G) Fold change of mRNA for Rack1 and p21 in prelesional paw skin of $\mathrm{Krt16}^{-/-}$mice relative to WT. Data represent mean \pm SEM of 4 biological replicates. Student's $t$ test. (H) Schematic of proposed regulation of NRF2 pathway in the suprabasal epidermis under normal physiological conditions and without K16. ${ }^{*} P<0.01$.

Fourth, we measured the levels of RACK1 protein in prelesional $\mathrm{Krt16}^{-\mathrm{p}}$ paw tissue in order to assess the physiological consequence of the lack of physical interaction between K16 and RACK1 uncovered in cultured keratinocytes. RACK1 is a known NRF2 target (28) and, accordingly, its levels should be depressed given the markedly hypoactive character of the latter in $\mathrm{Krt16}^{-/}$footpad skin. Relative to control skin, there was a marked reduction of
RACK1 protein, as detected by indirect immunofluorescence (Figure 5, D and E) and Western blotting (Figure 5F). Similarly to p-NRF2 (Ser-40) and its downstream targets, RACK1 was diminished in both the epidermis and the dermis of $\mathrm{Krt}^{-16^{-1}}$ skin (by 4.34 \pm 0.03 -fold and $2.9 \pm 0.10$-fold, respectively) (Figure $5 \mathrm{E}$ ). In contrast to the protein, Rack1 mRNA levels remained comparable to those of control (Figure 5G). 
A

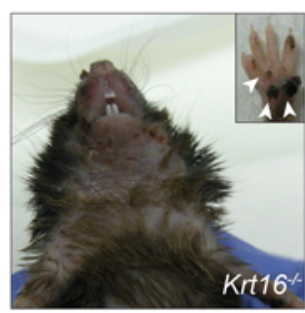

C
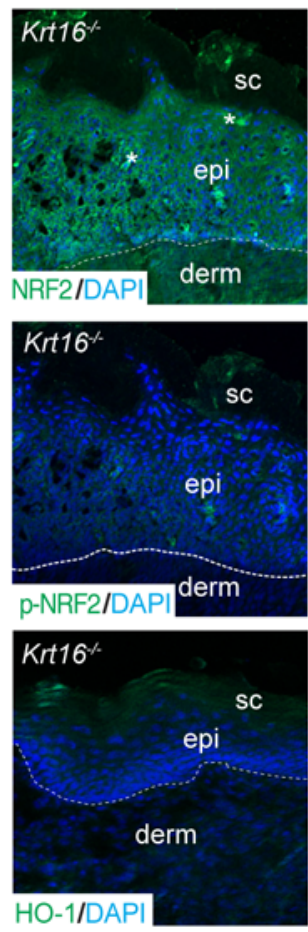

SF

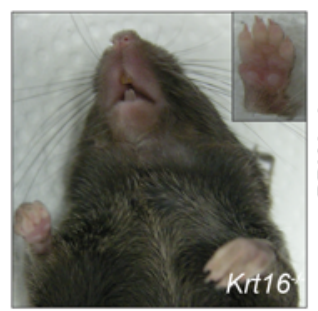

SF
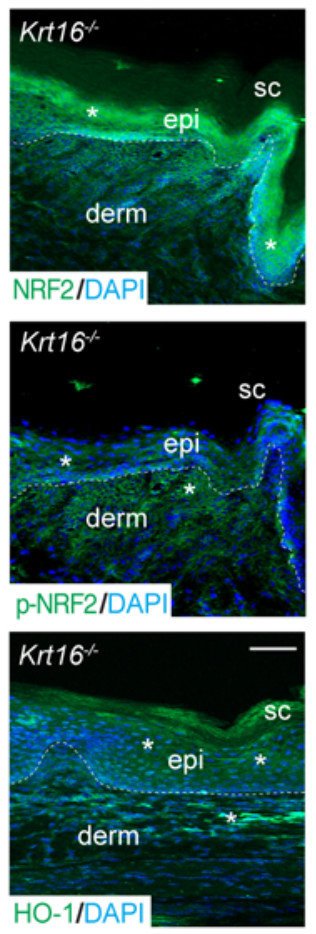

B
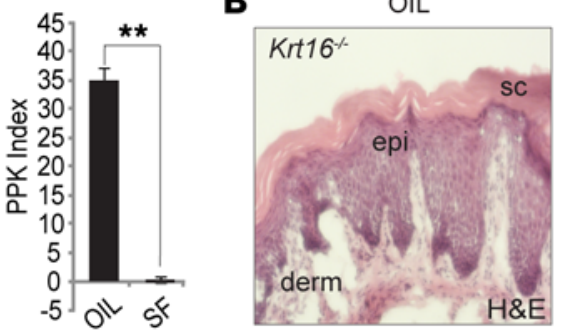

D

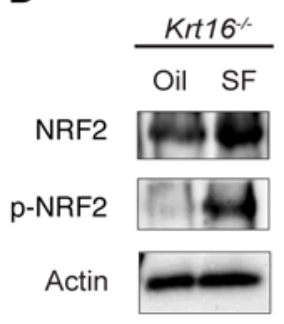

E

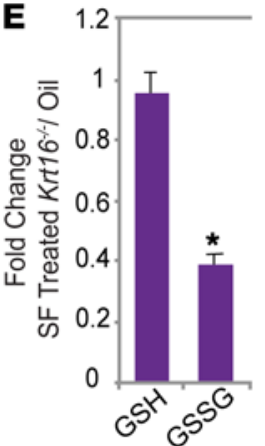

SF

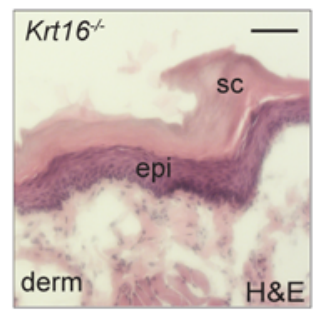

G

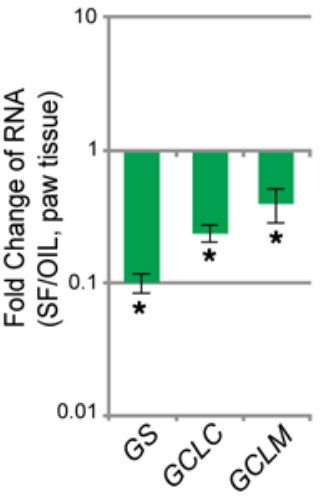

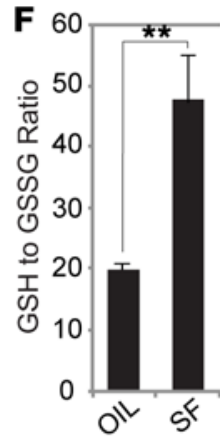

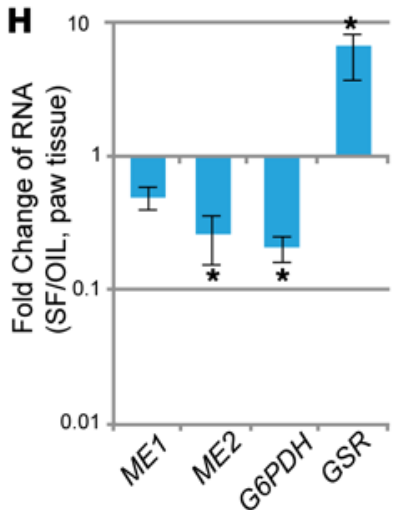

Figure 6. Treatment with SF prevents hyperkeratotic front paw calluses in $\mathrm{Krt16}^{-/-}$mice. (A) Representative images of $\mathrm{Krt16}^{-/-}$mice treated with SF or vehicle ( $n=10$ mice per group). Insets show representative close-ups of paws. Arrowheads depict areas of skin hyperpigmentation, reflecting lesions. Quantitation of PPK index is provided at right. (B) H\&E staining of SF- and jojoba oil-treated (vehicle) paw skin. Scale bar: $50 \mu \mathrm{m}$. (C) Indirect immunofluorescence for NRF2, p-NRF2, and HO-1. Dotted lines mark dermoepidermal junction. Scale bar: $50 \mu \mathrm{m}$. Original magnification, $\times 100$. Asterisks mark areas of increased indirect immunofluorescence. (D) Representative Western blots of NRF2 and p-NRF2 with actin as loading control. (E) Fold change in GSH and GSSG and (F) GSH to GSSG ratio for $\mathrm{Krt16}^{-/-}$paw skin treated with SF or vehicle. Data represent mean \pm SEM of 6 biological replicates. Student's $t$ test. (G and $\mathbf{H}$ ) Relative fold change in mRNA levels of GSH synthesis enzymes (G) and GSH metabolism enzymes (H). Data represent mean \pm SEM of 4 biological replicates involving 2-month-old male mice. Student's $t$ test. log scale. ${ }^{*} P<0.05 ;{ }^{* *} P<0.01$.

Another mechanism of interest regarding NRF2 activation in skin keratinocytes consists of the TGF- $\beta$-dependent transcriptional activation of $\mathrm{p} 21$, which was recently shown to stabilize NRF2 and enhance GSH metabolism (29). Interestingly, we found that p21 protein levels were decreased by 2 -fold in $\mathrm{Krt16}^{-/}$epidermis (Figure 5, D and E) and mRNA levels were decreased by 2-fold in total paw skin (Figure 5G). Protein levels of p-SMAD2, a key terminal effector of the TGF- $\beta$ signaling pathway, were also lower in $\mathrm{Krt16}^{--}$skin versus WT control (Figure 5F). Since overexpression of RACK1 in NIH 3T3 cells has been correlated with increased p21 expression (30) and RACK1 has been shown to both promote and be a target of TGF- $\beta 1$ signaling in fibrotic liver (31), the reduction in p21 levels and the impairment of TGF- $\beta$ signaling in $\mathrm{Krt16}^{--}$skin may be related to the decrease of RACK1. These results point to the existence of a connection among K16, TGF- $\beta$ signaling, RACK1 protein synthesis and/or stability, PKC $\delta$ activity, and $p 21$ transcription, all of which are capable of significantly affecting NRF2 activity in the suprabasal epidermis under stress conditions. We hypothesize that K16, either through a competitive effect on the sequestration of RACK1 by K14 and K5 or a still unclear mechanism, promotes the stabilization of RACK1, which ultimately results in NRF2 activation that is confined to the suprabasal layer (Figure $5 \mathrm{H}$ ).

Small molecule-based activation of NRF2 rescues PPK in $\mathrm{Krt16}^{-1-}$ mice. To further the relevance of NRF2's markedly attenuated activity in the development of the hyperkeratotic calluses in $\mathrm{Krt} 16^{-/-}$ mice, we topically treated 1-month-old male $\mathrm{Krt16}^{--}$mice with sulforaphane (SF), an NRF2 inducer derived from broccoli sprout extract (32), twice weekly for 4 consecutive weeks. This seemed a plausible approach given the high amounts of NRF2 protein avail- 
able for activation in $\mathrm{Krt16}^{-/-}$skin. Remarkably, significantly fewer hyperpigmented calluses were observed in SF-treated $\mathrm{Krt16}^{-/}$ paws (Figure 6A). A blinded assessment (Supplemental Figure 4) ascertained that the average affected surface area of SF-treated paws was dramatically lower $(0.08 \% \pm 0.07 \%$ in SF-treated versus $34.9 \% \pm 3.2 \%$ for vehicle-treated paws; mean \pm SEM; $n=10$ mice per experimental group) (Figure 6A). Histologically, the extent of epidermal thickening was markedly less in SF-treated paw tissue relative to vehicle treated (Figure 6B). As anticipated, increased indirect immunofluorescence signals for NRF2, p-NRF2 (Ser40), and $\mathrm{HO}-1$ occurred in both the epidermis and dermis of SF-treated $\mathrm{Krt}_{16} \mathrm{C}^{-/}$paws (Figure 6C). The increases in NRF2 and p-NRF2 were confirmed with Western blotting (Figure 6D). Indirect immunofluorescence signals for RACK1, PKC $\delta$, and p-PKC $\delta$ were also increased in the epidermis of $\mathrm{Krt16}^{-/}$paws following SF treatment (Supplemental Figure 5). These results suggest that topical treatment with SF successfully activated NRF2 in $\mathrm{Krt}^{-/-}$paw skin tissue. Strikingly, GSH levels were similar in $\mathrm{Krt16}^{-/-}$mice whether they were treated with SF or vehicle $(0.96 \pm 0.07$-fold change; mean \pm SEM); however, GSSG levels were diminished $2.56 \pm$ 0.05 -fold following SF treatment (Figure 6E; mean \pm SEM). This resulted in decreased oxidative stress overall, as indicated by a higher GSH to GSSG ratio (47.1 \pm 7.8 in SF-treated versus $19.9 \pm 0.9$ in vehicle-treated $\mathrm{Krt16}^{-/-}$paw tissue) (Figure 6F; mean $\pm \mathrm{SEM}$ ). After SF treatment, the mRNA levels of Gss, Gclc, Gclm, Me1/2, and G6pd were down, but there was a significant, $6.6 \pm 2.4$-fold, rise in Gsr mRNA (Figure 6, G and H; mean \pm SEM). Taken together, these unexpected findings imply that topical treatment with $\mathrm{SF}$ improves regeneration of GSH, but GSH synthesis remains defective in skin lacking K16.

\section{Discussion}

We previously reported that $\mathrm{Krt}_{1} \mathrm{6}^{-/-}$mice develop footpad lesions that histologically and molecularly mimic PC-associated PPK (13). In the present study, we determined that there is increased oxidative stress associated with an impairment of GSH synthesis, prior to lesion onset, in $\mathrm{Krt16}^{-/-}$paw skin. This oxidative stress, by itself or as a trigger of cell/tissue damage, is poised to play a significant role in the phenomenon of increased expression of DAMPs and innate immunity markers. It may also stimulate regulators of the skin barrier in $\mathrm{Krt16}^{-/}$paw skin (11) and originates, to a substantial degree, from the dysfunction of the KEAP1/NRF2 signaling pathway. In vitro and in vivo experiments revealed a connection between Krt16 expression and activation of NRF2, as assessed via nuclear localization, phosphorylation status, and expression of key target genes. Two important upstream regulators of NRF2 activity, RACK1 and PKC $\delta$, were found to be hypoactive and/or decreased in $\mathrm{Krt16}^{-/-}$paw skin. Genetic ablation of $\mathrm{Nrf2}$ resulted in an earlier onset of PPK lesions in $\mathrm{Krt16}^{-/-}$mice, whereas topical treatment with the NRF2 activator SF prevented lesion formation and restored redox balance to near normal in $\mathrm{Krt16}^{-/-}$mice, confirming the significance of the NRF2 pathway in the pathophysiology of hyperkeratotic paw calluses. Finally, we provided evidence that NRF2 can be readily detected but is hypophosphorylated, reflecting low activity, in lesional plantar skin tissue of individuals with PC, lending strong support to the relevance and significance of the observations made in the Krt16-null mouse model.
Our results suggest a role for K16 in defining the skin's response to oxidative stress through the modulation of the NRF2 signaling pathway. NRF2 is constitutively expressed in keratinocytes of normal skin and is upregulated upon wounding and exposure to electrophiles (33). Following UVB irradiation, NRF2 activates the production and export of GSH by suprabasal keratinocytes, which subsequently protect basal keratinocytes in a paracrine manner (34). We show here that the loss of Krt16, whose expression in glabrous skin is normally limited to the suprabasal compartment, selectively affects GSH production. Thus, rather than a global impact on NRF2 signaling, K16 appears to regulate a subset of the NRF2 transcriptional program. Unlike the basal-specific keratins K5 and K14, K16 does not appear to sequester RACK1 and subsequently inhibit PKC activation. In the absence of K16, in fact, RACK1 protein levels and $\mathrm{p}-\mathrm{PKC} \delta$ are markedly lower in glabrous skin. TGF- $\beta$ signaling through SMAD2 is also impaired, and levels of p21 are reduced in skin lacking K16. Taken together, these findings imply that K16, whose expression can be stimulated by TGF- $\beta$ in cultured keratinocytes (35), may be involved in a complex signaling network involving TGF- $\beta$, p21, RACK1, and NRF2 that ultimately affects redox balance in skin keratinocytes. The positive impact of K16 on TGF- $\beta$ signaling may, under normal conditions, contribute to the suprabasal-to-basal gradient of NRF2 (and its target genes) brought to light by Schafer et al. following UVB irradiation (34). This suggests that K16 is poised to be a key contributor to the unique cellular redox capabilities of the suprabasal compartment of epidermis in glabrous skin. Interestingly, Krt16 is an NRF2 target gene $(19,21)$. The role of K16 toward NRF2 activation shown in this study suggests that it powers a positive feedback loop toward the regulation of NRF2 function in skin keratinocytes. We recently showed that the related $\mathrm{K} 17$ acts in a similar fashion with regard to the expression of proinflammatory cytokines in tumor-prone skin keratinocytes, achieved through its interaction with the ribonucleoprotein heterogeneous nuclear ribonucleoprotein K (hnRNP K) (36).

Our findings extend the current understanding of the pathogenesis of the painful and debilitating PPK associated with PC and provide compelling evidence that the noncanonical functions of keratins represent therapeutic targets for keratin-based disorders. We provide evidence in the current study that NRF2 function is likely compromised in lesional PPK-stricken plantar skin tissue from individuals with PC. Whether alterations in GSH metabolism and oxidative stress occur in the setting of PPK in individuals with PC and whether these findings are also relevant to the pathogenesis of related skin disorders are open issues of great significance. Besides, current treatment strategies for PC focus on silencing of the affected keratin genes and have yielded mixed results (37-40). Simply silencing mutant keratin gene expression may be insufficient for an effective treatment of PC, and at the very least, a combination of gene silencing and a pharmacological restoration of redox balance and NRF2 signaling may be required to achieve a significant clinical improvement of PPK. SF, particularly in its natural form as broccoli sprout extract, is clinically attractive as a pharmacological NRF2 inducer given several previous studies demonstrating the safety of topical use in murine and human skin (41-43). On a different level, SF could exert NRF2-independent effects that contribute to the rescue of the $\mathrm{Krt}_{16^{-/-}}$mice, a possibility that deserves 
further investigation and that may add to the clinical benefit of the application of SF in PC. Also of interest, the antioxidant effects of NRF2 extend well beyond GSH metabolism and also include, for instance, a stimulation of mitochondrial biogenesis, metabolism, and function $(44,45)$. Such elements may contribute to the pathogenesis of PPK lesions and as such represent additional therapeutic targets of interest in PC and related disorders.

In a broader context, an enhanced understanding of the pathophysiology of PC opens up new therapeutic avenues for more common deficiencies in wound healing that plague clinical practice today. Given that it arises from mutations in wound-inducible keratins and is associated with an impairment of cellular stress responses, PC possibly represents a genetically determined disorder in which wound healing is markedly impaired. The lessons learned regarding the role of keratins in the regulation of redox balance in the course of investigating PC may be applicable to additional conditions in which oxidative stress plays a key role, e.g., diabetic wound ulcers and nonhealing chronic wounds. Pharmacological manipulation of redox balance and/or keratin expression may also provide a viable treatment approach for these challenging maladies.

\section{Methods}

Animals, antibodies, and preparation of SF. $\mathrm{Krt16}^{-/-}$and $\mathrm{Nrf2}^{-{ }_{-1}}$ mouse lines on a C57BL/6 background were maintained under specific pathogen-free conditions and fed chow and water ad libitum. $\mathrm{Krt16}^{-/-}$mice were generated by our laboratory (13). $N r f 2^{-/-}$mice were provided by Thomas Kensler (Johns Hopkins University). Commercial antibodies used are listed in Supplemental Table 1. Generation of the K16 antibody has been described (46). Alexa Fluor-conjugated secondary antibodies as well as goat anti-mouse and goat anti-rabbit (Life Technologies) antibodies were also used. SF was obtained from LKT Laboratories Inc. Stock solutions of SF were prepared at $0.1 \mathrm{M}$ in DMSO vehicle. For topical application, SF was diluted to the desired final concentration in jojoba oil (MP Biomedicals LLC) on the day of the treatment, as described $(19,43)$.

Mouse and cell culture studies. Four- and 6-week-old $\mathrm{Krt16}^{-/-}$or WT male mice were topically treated with either $100 \mu \mathrm{l}$ of $1 \mu$ mole SF or vehicle control on their front paws twice weekly for 4 weeks. Upon completion of the treatment regimen, the ventral surface of each treated paw was photographed, and the extent of PPK-like lesions was blindly and independently assessed by 5 individuals as described in Supplemental Figure 2.

Four-week-old WT mice were given i.p. injections of $50 \mu$ mole BSO (Sigma-Aldrich), an inhibitor of GSH synthesis, in PBS or PBS alone twice weekly (19). The mice were harvested after 4 weeks; levels of GSH and GSSG levels in paw tissue were assessed, and histological analysis was performed.

The 308 mouse epidermal keratinocytes (47) were cultured in DMEM (Invitrogen) containing 10\% FBS (Atlanta Biologicals), 100 units $/ \mathrm{ml}$ penicillin, and $100 \mu \mathrm{g} / \mathrm{ml}$ streptomycin (Invitrogen) at $37^{\circ} \mathrm{C}$ in $5 \% \mathrm{CO}_{2}$. GFP-K16 was transiently transfected using FuGENE HD transfection reagent (Promega) according to the manufacturer's protocol. Indirect immunofluorescence was performed as described (48). GFPK16- or GFP-K14-transfected 308 mouse epidermal keratinocytes were fixed in $4 \%$ paraformaldehyde for 10 minutes at room temperature, washed in PBS, blocked in 5\% normal goat serum/0.1\% Triton X-100/ $\mathrm{PBS}$ for 1 hour at room temperature, incubated in primary antibody solution for 1 hour, washed in PBS, incubated in secondary antibody solution for 1 hour, counterstained with DAPI, and mounted in FluorSave Reagent Mounting Medium (EMD Millipore) before visualization using a Zeiss fluorescence microscope with an ApoTome attachment.

Human studies. Punch biopsies $(3 \mathrm{~mm})$ from PC patients were obtained as previously described (48) and analyzed for protein expression by immunohistochemistry. Cryosections $(10 \mu \mathrm{m})$ were fixed with ice-cold acetone for 20 minutes, washed with PBS, and incubated with $2 \%$ BSA in PBS for 1 hour at room temperature to minimize nonspecific binding. The sections were incubated overnight at $4^{\circ} \mathrm{C}$ in primary antibody solution, washed in PBS, and incubated in secondary antibody solution for 1 hour. Following counterstaining with DAPI and mounting in Hydromount (National Diagnostics), protein expression was visualized using a Zeiss fluorescence microscope.

Biochemical and morphological analysis. RNA was extracted from front paws using TRIzol reagent (Life Technologies). After DNAse treatment (RNAse free DNase kit: QIAGEN), $1 \mu \mathrm{g}$ of total RNA from each sample was reverse transcribed (iScript cDNA synthesis kit; BioRad). Quantitative RT-PCR was performed as described (49). The target-specific oligonucleotide primer sets used are listed in Supplemental Table 2. For protein analyses, paw tissue was homogenized and extracted using TRIzol reagent (Life Technologies). Protein concentration was measured using a Bradford Assay Kit (Bio-Rad). Equal amounts of protein (15-20 $\mu \mathrm{g}$ range) were loaded onto 10\% SDS/PAGE gels and blotted onto nitrocellulose. Actin was used as a loading control in all immunoblots. Bound primary antibodies were detected with enhanced chemiluminescence (Thermo Scientific). GSH and GSSG levels in skin tissue were assessed using a Glutathione Fluorometric assay kit from BioVision. NADPH levels were measured using a colorimetric kit (BioVision). For histological analyses, paw tissue samples were submerged in OCT (Sakura Finetek), flash-frozen in liquid nitrogen, and stored at $-20^{\circ} \mathrm{C}$ upon sectioning. Sections of $8 \mu \mathrm{m}$ were cut in a specific and consistent orientation relative to paw morphology and stained with either H\&E for routine histopathology or incubated with primary antibodies and Alexa Fluor-conjugated secondary antibodies for indirect immunofluorescence (49).

Statistics. Unpaired 2-tailed Student's $t$ test and ANOVA were performed when appropriate. Significant differences between 2 groups were noted by asterisks. Quantification data were presented in mean \pm SEM. Blinded quantitation of indirect IF was performed using Image (NIH).

Study approval. All studies involving mice were approved by the Johns Hopkins University Institutional Animal Care and Use Committee. Human plantar skin biopsies were obtained using standard surgical techniques with patient consent under W-IRB \#2004/0468/1057496 (47).

\section{Author contributions}

MLK, JMCH, RGL, and YG conducted all experiments and assisted with the analysis and interpretation of the data. MLK and PAC designed the experiments and wrote the manuscript. $\mathrm{AB}$ and RLK played a key role in generating the human data. All authors read and commented on the manuscript.

\section{Acknowledgments}

The authors thank Mary Schwartz and David C. Hansen from the Pachyonychia Congenita Project (www.pachyonychia.org) and members of the Coulombe laboratory for support; PC patients and nonpatient volunteers for providing biopsies; Michael Polydefkis, 
Baohan Pan, and Madelyn Low for assistance; Christopher Contag for support; and Juliane Lessard and Tom Kensler for advice. This work was supported by R01 grant AR44232 (to P.A. Coulombe) from the NIH. M.L. Kerns received support from T32 training grant CA009110 from the NIH.
Address correspondence to: Pierre A. Coulombe, Department of Biochemistry and Molecular Biology, Johns Hopkins Bloomberg School of Public Health, 615 N. Wolfe Street, Room W8041, Baltimore, Maryland 21205, USA. Phone: 410.955.3671; E-mail: coulombe@jhu.edu.
1. Coulombe PA, Hutton ME, Vassar R, Fuchs E. A function for keratins and a common thread among different types of epidermolysis bullosa simplex diseases. J Cell Biol. 1991;115(6):1661-1674.

2. Omary MB, Coulombe PA, McLean WH. Intermediate filament proteins and their associated diseases. NEngl JMed. 2004;351(20):2087-2100.

3. Szeverenyi I, et al. The Human Intermediate Filament Database: comprehensive information on a gene family involved in many human diseases. Hum Mutat. 2008;29(3):351-360.

4. Coulombe PA, Kerns ML, Fuchs E. Epidermolysis bullosa simplex: a paradigm for disorders of tissue fragility. J Clin Invest. 2009;119(7):1784-1793.

5. Pan X, Hobbs RP, Coulombe PA. The expanding significance of keratin intermediate filaments in normal and diseased epithelia. Curr Opin Cell Biol. 2013;25(1):47-56.

6. Hamada T, et al. How do keratinizing disorders and blistering disorders overlap? Exp Dermatol. 2013;22(2):83-87.

7. Smith FJ, et al. The genetic basis of pachyonychia congenita. J Invest Dermatol Symp Proc. 2005;10(1):21-30.

8. McLean WH, et al. Keratin 16 and keratin 17 mutations cause pachyonychia congenita. Nat Genet. 1995;9(3):273-278.

9. McGowan K, Coulombe PA. The wound repairassociated keratins 6,16, and 17. Subcell Biochem. 1998;31:173-204.

10. Eliason MJ, Leachman SA, Feng BJ, Schwartz ME, Hansen CD. A review of the clinical phenotype of 254 patients with genetically confirmed pachyonychia congenita. J Am Acad Dermatol. 2012;67(4):680-686.

11. Lessard JC, et al. Keratin 16 regulates innate immunity in response to epidermal barrier breach. Proc Natl Acad Sci U S A. 2013;110(48):19537-19542.

12. Shah S, Boen M, Kenner-Bell B, Schwartz M, Rademaker A, Paller AS. Pachyonychia congenita in pediatric patients: natural history, features, and impact. JAMA Dermatol. 2014;150(2):146-153.

13. Lessard JC, Coulombe PA. Keratin 16-null mice develop palmoplantar keratoderma, a hallmark feature of pachyonychia congenita and related disorders. J Invest Dermatol. 2012;132(5):1384-1391.

14. Lushchak VI. Glutathione homeostasis and functions: potential targets for medical interventions. J Amino Acids. 2012;2012:736837.

15. Pastore A, et al. Determination of blood total, reduced, and oxidized glutathione in pediatric subjects. Clin Chem. 2001;47(8):1467-1469.

16. Dalton TP, Dieter MZ, Yang Y, Shertzer HG, Nebert DW. Knockout of the mouse glutamate cysteine ligase catalytic subunit (Gclc) gene: embryonic lethal when homozygous, and proposed model for moderate glutathione deficiency when heterozygous. Biochem Biophys Res Commun. 2000;279(2):324-329.
17. Yang Y, Dieter MZ, Chen Y, Shertzer HG, Nebert DW, Dalton TP. Initial characterization of the glutamate-cysteine ligase modifier subunit Gclm(-/-) knockout mouse. J Biol Chem. 2002;277(51):49446-49452.

18. Couto N, Malys N, Gaskell SJ, Barber J. Partition and turnover of glutathione reductase from Saccharomyces cerevisiae: a proteomic approach. J Proteome Res. 2013;12(6):2885-2894.

19. Kerns M, DePianto D, Yamamoto M, Coulombe PA. Differential modulation of keratin expression by sulforaphane occurs via Nrf2-dependent and -independent pathways in skin epithelia. Mol Biol Cell. 2010;21(23):4068-4075.

20. Gorrini C, Harris IS, Mak TW. Modulation of oxidative stress as an anticancer strategy. Nat Rev Drug Discov. 2013;12(12):931-947.

21. Endo H, Sugioka Y, Nakagi Y, Saijo Y, Yoshida T. A novel role of the NRF2 transcription factor in the regulation of arsenite-mediated keratin 16 gene expression in human keratinocytes. Environ Health Perspect. 2008;116(7):873-879.

22. Alam J, Cook JL. Transcriptional regulation of the heme oxygenase-1 gene via the stress response element pathway. Curr Pharm Des. 2003;9(30):2499-2511.

23. Kwak MK, Itoh K, Yamamoto M, Sutter TR, Kensler TW. Role of transcription factor Nrf2 in the induction of hepatic phase 2 and antioxidative enzymes in vivo by the cancer chemoprotective agent, 3H-1, 2-dimethiole-3-thione. Mol Med. 2001;7(2):135-145.

24. Itoh K, et al. Keap1 represses nuclear activation of antioxidant responsive elements by Nrf 2 through binding to the amino-terminal Neh2 domain. Genes Dev. 1999;13(1):76-86.

25. Niture SK, Jain AK, Jaiswal AK. Antioxidantinduced modification of INrf2 cysteine 151 and PKC-delta-mediated phosphorylation of Nrf2 serine 40 are both required for stabilization and nuclear translocation of Nrf2 and increased drug resistance. J Cell Sci. 2009;122(pt 24):4452-4464

26. Adams DR, Ron D, Kiely PA. RACK1, A multifaceted scaffolding protein: structure and function. Cell Commun Signal. 2011;9:22.

27. Kroger C, Loschke F, Schwarz N, Windoffer R, Leube RE, Magin TM. Keratins control intercellular adhesion involving PKC- $\alpha$-mediated desmoplakin phosphorylation. JCell Biol. 2013;201(5):681-692.

28. Kim YH, Coon A, Baker AF, Powis G. Antitumor agent PX-12 inhibits HIF-1 $\alpha$ protein levels through an Nrf2/PMF-1-mediated increase in spermidine/spermine acetyl transferase. Cancer Chemother Pharmacol. 2011;68(2):405-413.

29. Oshimori N, Oristian D, Fuchs E. TGF- $\beta$ promotes heterogeneity and drug resistance in squamous cell carcinoma. Cell. 2015;160(5):963-976.

30. Hermanto U, Zong CS, Li W, Wang LH. RACK1, an insulin-like growth factor I (IGF-I) receptor- interacting protein, modulates IGF-I-dependent integrin signaling and promotes cell spreading and contact with extracellular matrix. Mol Cell Biol. 2002;22(7):2345-2365.

31. Jia D, et al. Up-regulation of RACK1 by TGF- $\beta 1$ promotes hepatic fibrosis in mice. PLoS One. 2013;8(3):e60115.

32. Zhang Y, Talalay P, Cho CG, Posner GH. A major inducer of anticarcinogenic protective enzymes from broccoli: isolation and elucidation of structure. Proc Natl Acad Sci U S A. 1992;89(6):2399-2403.

33. Braun S, et al. Nrf2 transcription factor, a novel target of keratinocyte growth factor action which regulates gene expression and inflammation in the healing skin wound. Mol Cell Biol. 2002;22(15):5492-5505.

34. Schafer M, Dutsch S, auf dem Keller U, Werner S. Nrf2: a central regulator of UV protection in the epidermis. Cell Cycle. 2010;9(15):2917-2918.

35. Choi Y, Fuchs E. TGF- $\beta$ and retinoic acid: regulators of growth and modifiers of differentiation in human epidermal cells. Cell Regul. 1990;1(11):791-809.

36. Chung BM, Arutyunov A, Ilagan E, Yao N, WillsKarp M, Coulombe PA. Regulation of C-X-C chemokine gene expression by keratin 17 and hnRNP K in skin tumor keratinocytes. JCell Biol. 2015;208(5):613-627.

37. Goldberg I, Fruchter D, Meilick A, Schwartz ME, Sprecher E. Best treatment practices for pachyonychia congenita. J Eur Acad Dermatol Venereol. 2014;28(3):279-285.

38. Leachman SA, et al. First-in-human mutationtargeted siRNA phase $\mathrm{Ib}$ trial of an inherited skin disorder. Mol Ther. 2010;18(2):442-446.

39. Milstone LM, et al. Treatment of pachyonychia congenita. J Investig Dermatol Symp Proc. 2005;10(1):18-20.

40. Trochet D, Prudhon B, Vassilopoulos S, Bitoun M. Therapy for dominant inherited diseases by allele-specific RNA interference: successes and pitfalls. Curr Gene Ther. 2015;15(5):503-510.

41. Kerns ML, DePianto D, Dinkova-Kostova AT, Talalay P, Coulombe PA. Reprogramming of keratin biosynthesis by sulforaphane restores skin integrity in epidermolysis bullosa simplex. Proc Natl Acad Sci U S A. 2007;104(36):14460-14465.

42. Dinkova-Kostova AT, et al. Protection against UV-light-induced skin carcinogenesis in SKH-1 high-risk mice by sulforaphane-containing broccoli sprout extracts. Cancer Lett. 2006;240(2):243-252.

43. Dinkova-Kostova AT, et al. Induction of the phase 2 response in mouse and human skin by sulforaphane-containing broccoli sprout extracts. Cancer Epidemiol Biomarkers Prev. 2007;16(4):847-851.

44. MacGarvey NC, Suliman HB, Bartz RR, Fu P, Withers CM, KE Piantadosi CA. Activation of 


\section{RESEARCH ARTICLE}

mitochondrial biogenesis by heme-oxygenase1-mediated NF-E2-related factor-2 induction rescues mice from lethal Staphylococcus aureus sepsis. Am J Respir Crit Care Med. 2012;185(8):851-861.

45. Tufekci KU, Civi Bayin E, Genc S, Genc K. The Nrf2/ARE pathway: a promising target to counteract mitochondrial dysfunction in Parkinson's disease. Parkinsons Dis. 2011;2011:314082.

46. Bernot KM, Coulombe PA, McGowan KM. Keratin 16 expression defines a subset of epithelial cells during skin morphogenesis and the hair cycle. J Invest Dermatol. 2002;119(5):1137-1149.

47. Strickland JE, et al. Development of murine epidermal cell lines which contain an activated rasHa oncogene and form papillomas in skin
The Journal of Clinical Investigation

grafts on athymic nude mouse hosts. Cancer Res. 1988;48(1):165-169.

48. Seegmiller BL. Gene expression profiling in pachyonychia congenita skin. J Dermatol Sci. 2015;77(3):156-165.

49. Hobbs RP, et al. Keratin-dependent regulation of Aire and gene expression in skin tumor keratinocytes. Nat Genet. 2015;47(8):933-938. 